Supporting Information for

\title{
New tools for characterizing Metallic Nanoparticles: AgNPs, a case study
}

\author{
Rodrigo A. González-Fuenzalida, Yolanda Moliner-Martínez, Carmen Molins- \\ Legua, Vanesa Parada-Artigues, Jorge Verdú-Andrés. Pilar Campins-Falcó* \\ MINTOTA research group. Departament de Química Analítica, Facultat de Química, Universitat de \\ Valencia, C/ Doctor Moliner, 50, 46100 Burjassot, Valencia, Spain \\ *Corresponding authors: E-mail: pilar.campins@uv.es, rodrigo.gonzalez@uv.es; Fax:(+)34963543447
}

General Information. Experimental methods, mathematical procedures, complementary studies, optimizations as well as NPs characterization data (TEM pictures and histograms, UV-Vis spectra, Cap-LC and IT-SPME-Cap-LC chromatograms) are presented in the following sections.

\section{Section 1. Experimental section: reagents and synthesis methods.}

Reagents. Silver nitrate $(>99 \%$, Sigma-Aldrich), trisodium citrate (Guinama), potassium bromide (Sigma-Aldrich), hydrogen peroxide (Panreac), sodium borhydride (Fluka) and glycerol (Sigma-Aldrich) were used for making AgNPs. Sodium dodecyl sulfate (SDS, $>99 \%$, Merck), ammonium acetate (98\%, Sigma-Aldrich) and sodium thiosulfate (Merck) were used for preparing the Cap-LC mobile phase. 5, 20 and $40 \mathrm{~nm}$ AuNPs in $0.1 \mathrm{mM}$ PBS (phosphate buffered saline) solutions (Sigma-Aldrich) were used as reference standards. Ethanol (Romil), nitric acid (Panreac), benzalkonium chloride and cetrimonium bromide (BZKC and CTAB, Sigma-Aldrich) were used for additional tests on NPs. Water was purified through a Barnstead NANOpure II system.

AgNPs were synthesized using two different methods. The production rate of AgNPs was quantified using TMB (3,3',5,5'-tetramethylbenzidine) and its oxidative reaction with silver ions [1]. Free silver was not detected and thus complete conversion was achieved.

Frank's approach. Silver nanoparticles are prepared by reducing silver ions $\left(\mathrm{Ag}^{+}\right)$with sodium borohydride in presence of citrate anions [2]. To control the sizes and shapes of the resulting particles, hydrogen peroxide can be added to the mixture [3]. Briefly, trisodium citrate $(1.3 \mathrm{~mL}, 1 \% \mathrm{w} / \mathrm{v}), \mathrm{AgNO}_{3}(0.064 \mathrm{~mL}, 1 \% \mathrm{w} / \mathrm{v}), \mathrm{H}_{2} \mathrm{O}_{2}(0.1 \mathrm{~mL}, 30 \%$ $\mathrm{v} / \mathrm{v})$, and optionally $\mathrm{KBr}(0.086 \mathrm{~mL}, 1 \mathrm{mM})$ and $\mathrm{NaBH}_{4}(0.94 \mathrm{~mL}, 0.1 \% \mathrm{w} / \mathrm{v})$ were subsequently added in a $20 \mathrm{~mL}$ flask and then, diluted with water to obtain a final volume of $5 \mathrm{~mL}$. The reaction mixture was stirred for 15 minutes at room temperature. 
Finally, the samples were stored and kept in the dark after being diluted with water $(1: 8$ dilutions).

Changing the agitation time, the volume of water in the final dilution step and using (or not using) hydrogen peroxide as previously indicated are the factors responsible for a specific final average particle diameter [4]. When $\mathrm{KBr}$ was added to the mixture, triangular-shaped AgNPs were synthesized (silver nanoprisms). Otherwise, spherical nanoparticles were obtained.

Thermal synthesis. A variation of the classical Lee and Meisel method [5] which improves monodispersity was used. Briefly, a $50 \mathrm{ml}$ glycerol-water mixture $(40 \% \mathrm{v} / \mathrm{v}$ of glycerol) was stirred in a $100 \mathrm{~mL}$ flask and heated up to $95^{\circ} \mathrm{C}$. Then, $9 \mathrm{mg}$ of silver nitrate and, one minute later, $1 \mathrm{ml}$ of sodium citrate $(3 \%)$ were added to the solvent mixture. The reaction mixture was stirred for 1 hour at $95^{\circ} \mathrm{C}$. AgNPs were stored in a plastic centrifuge tube, once room temperature was reached.

\section{Section 2. Reagents and Instrumentation}

UV-visible spectra were registered with a Varian Cary 60 Fiber Optic UV-Vis spectrophotometer. The analytical signal was recorded between 200 and $1000 \mathrm{~nm}$. AgNP characterization was performed by using a transmission electronic microscope (TEM) with a JEM-1010 from Jeol Ltd. Operated at $100 \mathrm{kV}$. TEM samples were prepared by placing a drop $(\sim 10 \mu \mathrm{L})$ of the AgNP suspension (1:8 dilutions for all the sample, except 1:2 for $38.15 \mathrm{~nm}$ synthesis) onto a carbon-coated copper grid (300 mesh), which was subsequently dried overnight at room temperature. Silver nanoparticle sizes were calculated by using ImageJ and Histogram softwares. The concentration was estimated from TEM experiments as described in N.L. Pacioni et al. work [6]. Chromatographic studies were carried out using an Agilent Technologies High Performance Liquid Chromatography System equipped with a LC capillary binary gradient pump (Agilent 1200 Series), diode array detection and a $2 \mu \mathrm{L}$ injection volume valve (Cap-LC). Capillary columns of polydimethylsiloxane (PDMS) with 5\% and 35\% of polydiphenylsiloxane (TRB\%, Teknokroma) were used for In-Tube Solid-Phase Microextraction. The water used throughout all experiments was purified through a Barnstead NANOpure II system. For DLS analysis, a Zetasizer Nano ZS (Malvern) system to measure samples directly.

\section{Section 3. Estimation of AgNPs concentrations from TEM measurements}

With TEM measurements and an equation for estimating spherical gold nanoparticles from [6], the concentration of these nanoparticles ([AgNPs]) can be estimated using the formulas below ((1) and (2)), where $\mathrm{N}$ represents the ratio nanoparticle:atom, and $\left[\mathrm{Ag}^{+}\right]$the silver concentration in the sample. The NP diameter $\left(\mathrm{d}_{\mathrm{AgNPs}}\right)$ is an average obtained by counting a large number $(\mathrm{n}=500)$ of particles. For spherical silver nanoparticles, the number of atoms and, subsequently, concentration were calculated according to: 


$$
\begin{gathered}
\text { (1) } N=\left(\frac{d_{A g N P S}}{d_{A g}}\right)^{3} \\
\text { (2) }[A g N P]=\frac{\left[A g^{+}\right]}{N} \\
\text { (3) } d_{A g N P S}=\left(\frac{\left[A g^{+}\right] \cdot d_{A g}^{3}}{[A g N P S]}\right)^{1 / 3}
\end{gathered}
$$

The diameter of silver $\left(\mathrm{d}_{\mathrm{Ag}}\right)$ used was $0.289 \mathrm{~nm}$. Finally, the calculated [AgNPs] were corrected using the corresponding dilution factors. The dilution factor was 0.125 $(1: 8)$ or $0.0625(1: 16)$ for the syntheses prepared by Frank's approach. Thermal synthesis was 1:2 diluted.

In the reverse process, formulas can be used for estimating the NP diameter from the Cap-LC calculated and corrected (considering the dilution) concentration as well as (see equation (3)). The concentration, in turn, is obtained from the analytical signal (peak area) using the calibration curve.

\section{Section 4. UV-Vis signal and nanoparticle size relation}

Figure S1 shows the UV-Vis spectra for AgNP syntheses which had a similar surface plasmon resonance band, SPRB (but different TEM-measured $\mathrm{d}_{\mathrm{AgNPs}}$ ) and similar TEM-measured $\mathrm{d}_{\mathrm{AgNPs}}$ (but different SPRB). As it can be seen, no correlation between SPRB and $\mathrm{d}_{\mathrm{AgNPs}}$ (which can be related to [AgNPs]) could be established. UVVis measurements cannot be used for AgNP quantification as SPRB are affected not only by size, but also by shape and NP dispersion state.

a)

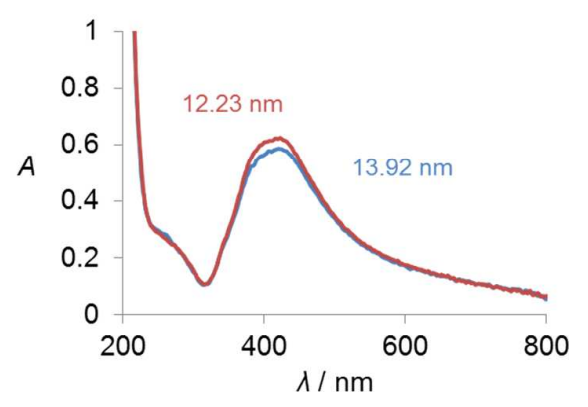

b)

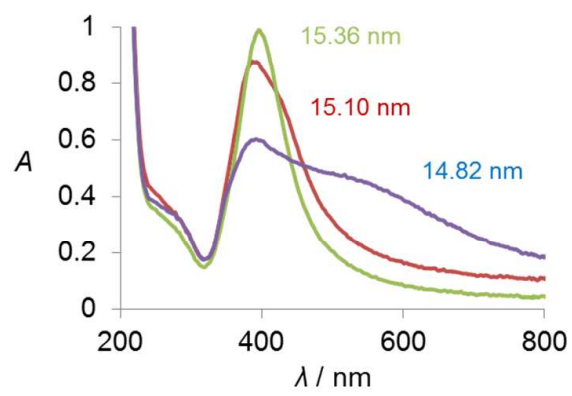

Figure S1. UV-Vis / TEM comparison. Absorption spectra of AgNPs with: (a) different and (b) similar average sizes. Cap-LC column effect. 


\section{Section 5. Optimization of the chromatographic conditions}

Figure S2 shows the chromatograms for the Cap-LC column, sodium thiosulfate concentration, and flow rate optimizations.

Different reversed phase Cap-LC columns: Zirchrom (Sachtopore-RP $5 \mu \mathrm{m}, 100$ $\AA$, $0.3 \mathrm{~mm}$ i.d. x $10 \mathrm{~cm})$, Zorbax SB-C18 $(5 \mu \mathrm{m}, 80 \AA$, $0.5 \mathrm{~mm}$ i.d. x $3.5 \mathrm{~cm})$, Zorbax SB-C18 $(3.5 \mu \mathrm{m}, 80 \AA, 0.5 \mathrm{~mm}$ i.d. x $3.5 \mathrm{~cm})$ and Jupiter C18 $(5 \mu \mathrm{m}, 300 \AA, 0.5 \mathrm{~mm}$ i.d. $\mathrm{x} 5 \mathrm{~cm}$ ) were tested. A $17.32 \mathrm{~nm} \mathrm{AgNP}$ synthesis (measured by TEM) prepared using Frank's approach was selected for performing the optimization study. For this study, the flow rate was fixed at $10 \mu \mathrm{L} \cdot \mathrm{min}^{-1}$. The best result was achieved when Jupiter C18 column was used (see Figure S2a).

As for sodium thiosulfate, three concentrations were tested: $0.5,1.0$ and $1.5 \mathrm{mM}$. The best signal profile was obtained for $1.0 \mathrm{mM}$ in terms of peak height and width (see Figure S2b). This reagent was added to the mobile phase for eluting by complexation $\mathrm{Ag}(\mathrm{I})$ species that could interact with the SDS-modified C18 surface and thereby affect the system conditions [7]. Although in the present work cationic silver conversions to AgNPs in the prepared syntheses were quantitative (confirmed by the TMB method) and NP oxidative dissolution to $\mathrm{Ag}^{+}$is not frequent [8], sodium thiosulfate was kept in the mobile phase as a precaution.

The flow rate was optimized: measurements were made at 5,10 and $20 \mu \mathrm{L} \cdot \min ^{-1}$ (the maximum operating value of the system pump). Chromatograms of AgNPs at the different flow rates are shown in Figure S2c. $10 \mu \mathrm{L} \cdot \mathrm{min}^{-1}$ was selected because the observed peak was both narrower and higher than that seen when working at 5 $\mu \mathrm{L} \cdot \mathrm{min}^{-1}$, and more column contact, and thus a higher signal, was present relative to working at $20 \mu \mathrm{L} \cdot \mathrm{min}^{-1}$. For all the flow rates, DAD spectra registered at least at three positions of the chromatographic peak showed the characteristic AgNP SPRB at 400 nm. 
a)

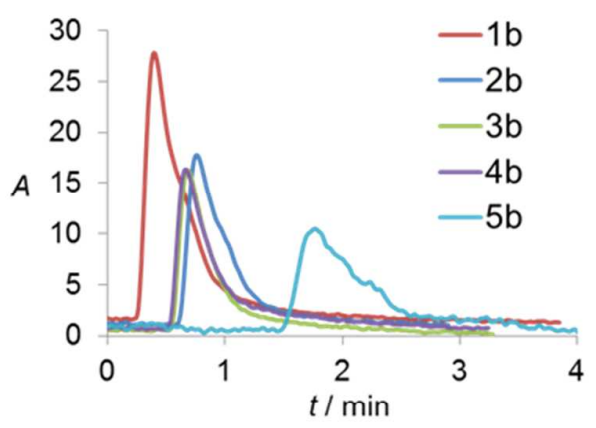

b)

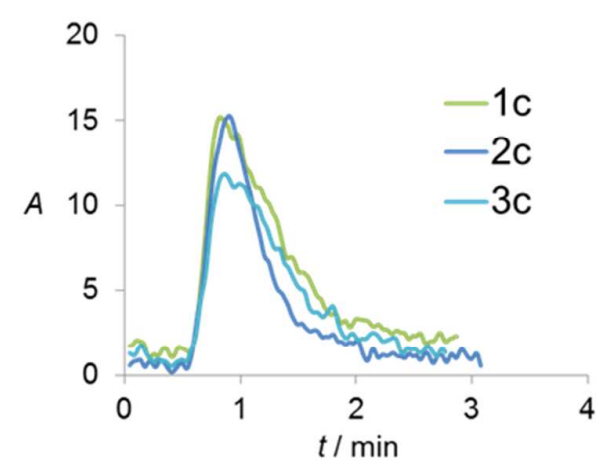

c)

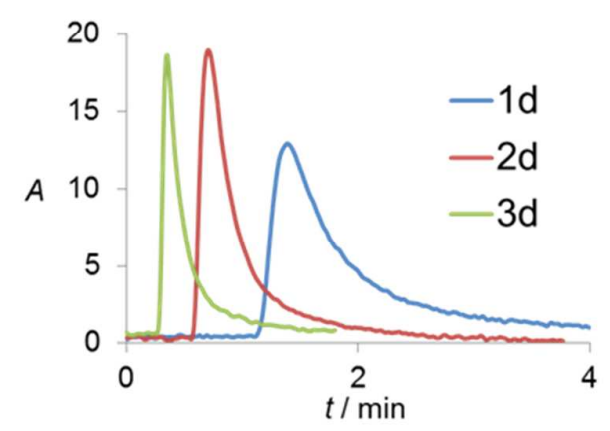

Figure S2. (a) AgNP chromatograms (1b) without and with: (2b) Jupiter column, (3b) $5 \mu \mathrm{m}$ particle size Zorbax column, (4b) $3.5 \mu \mathrm{m}$ particle size Zorbax and (5b) Zirchrom column. (b) AgNP Chromatograms with different $\mathrm{Na}_{2} \mathrm{~S}_{2} \mathrm{O}_{3}$ concentrations: (1c) $0.5 \mathrm{mM}$, (2c) $1.0 \mathrm{mM}$ and (3c) $1.5 \mathrm{mM}$. (c) AgNP chromatograms at different flow rates: (1d) 5 , (2d) 10 , and (3d) $20 \mu \mathrm{L} / \mathrm{min}$

\section{Section 6. Calibration curve and size estimations by Cap-LC}

The calibration curve obtained for the chosen standard synthesis $(17.32 \mathrm{~nm}$ AgNPs) is shown in Figure S3 (on the left). Also, another study was carried out to prove that the Cap-LC signal responded to AgNP concentration. An Area vs [AgNPs] representation at 3 levels of concentration $(0.21,0.42$ and $0.63 \mathrm{nM})$, each one containing 2 measures coming from different sized AgNPs, is represented in Figure S3 (on the right). A linear fit was obtained $\left(\mathrm{R}^{2}=0.995\right)$. 

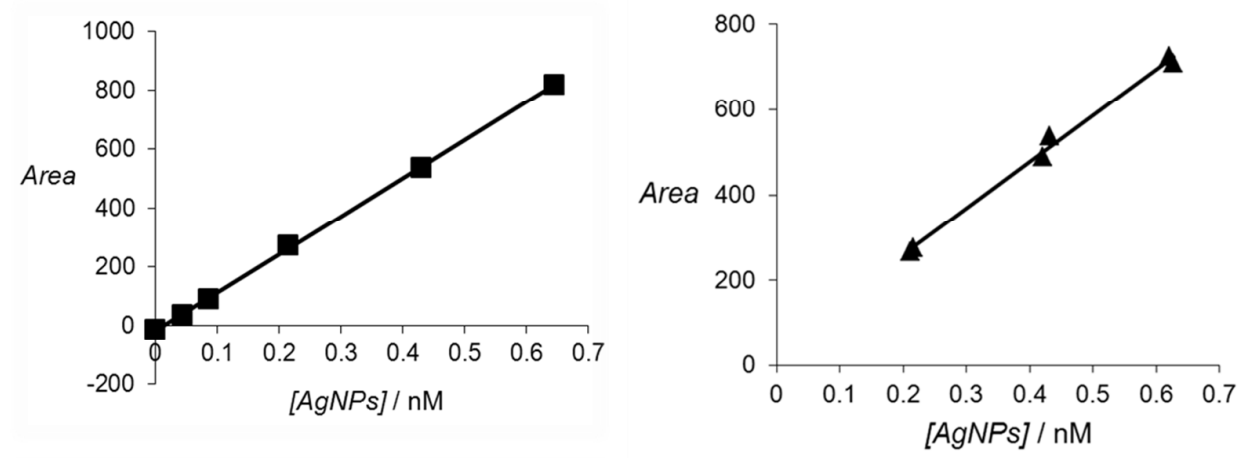

Figure S3. Representations of: (left) calibration curve for $17.32 \mathrm{~nm} \mathrm{AgNPs}$ and (right) Area vs [AgNPs] plot at 3 levels of concentration $(0.21,0.42$ and $0.63 \mathrm{nM})$ where each measure comes from different sized AgNP samples.

Section 7. UV-Vis spectra, TEM measurements and Cap-LC signals for AgNP syntheses:

\section{$17.32 \mathrm{~nm}$ Standard}

Synthesis was characterized by TEM (Figure S4), UV-Vis and Cap-LC (Figure S5). It was used as calibration standard and for optimization steps. 1:8 dilution of 17.32 $\mathrm{nm}$ AgNPs corresponds to $0.431 \mathrm{nM}$ of concentration.

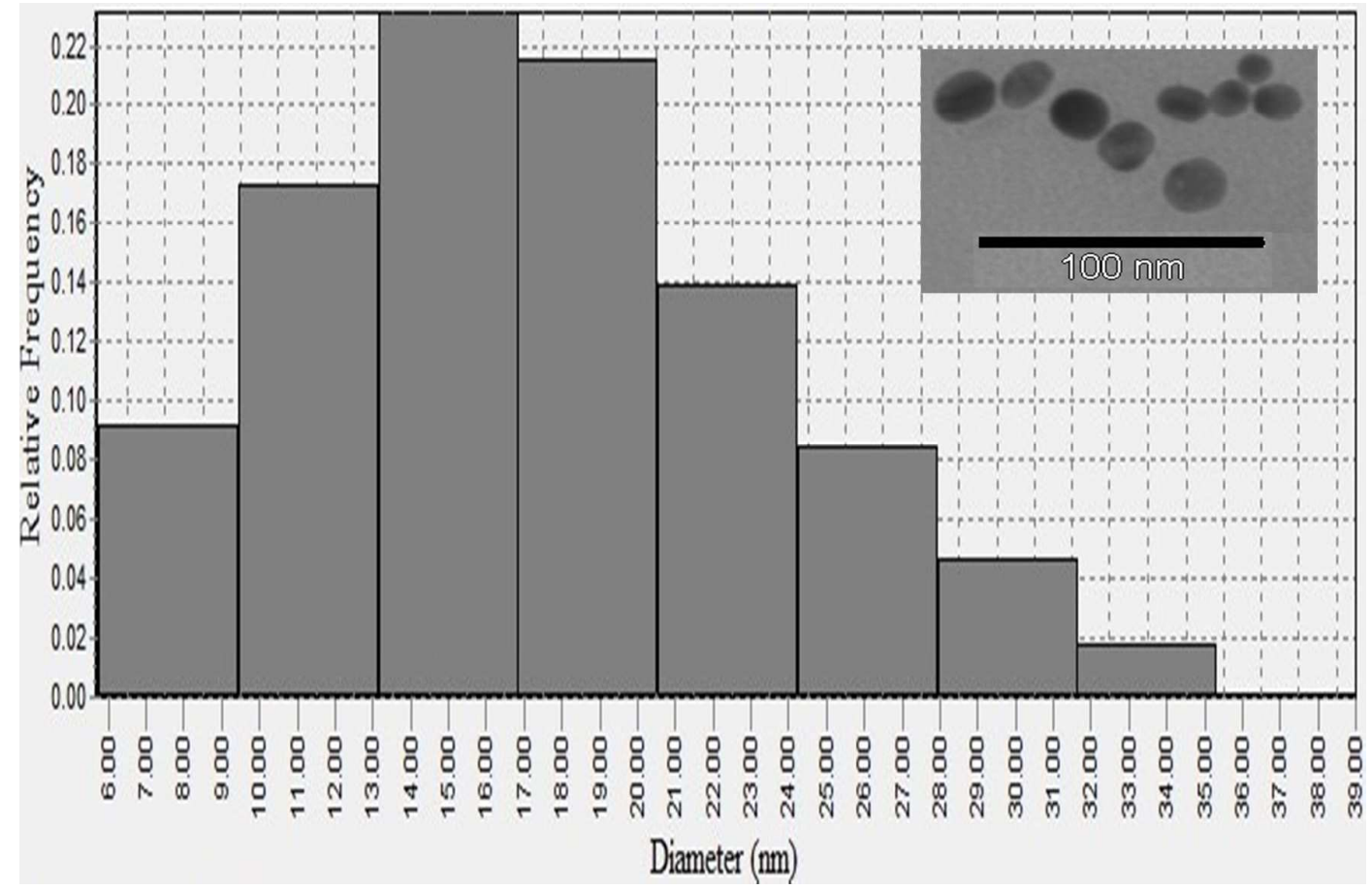

Figure S4. TEM histogram of $17.32 \mathrm{~nm}$ AgNPs showing their size distribution. Up-right: TEM picture. 

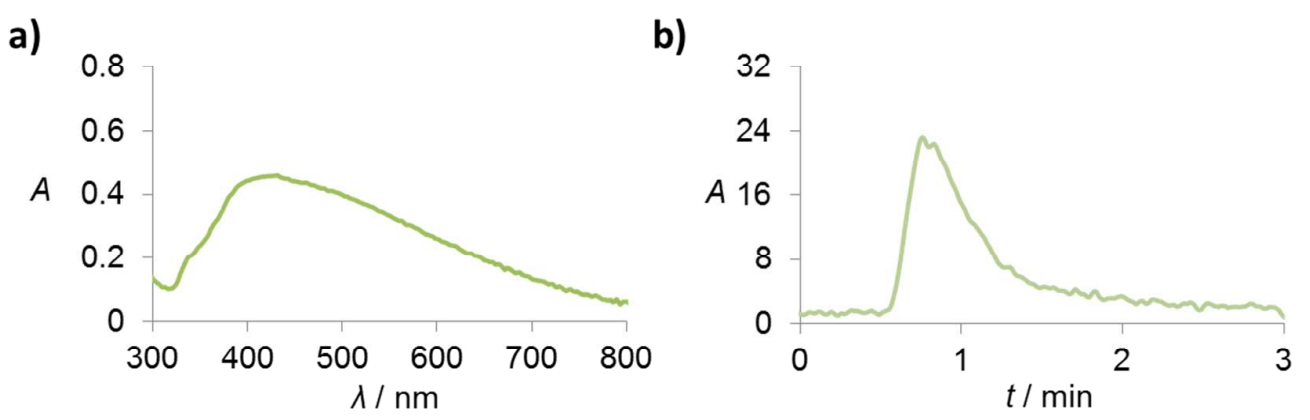

Figure S5. (a) UV-Vis spectrum of 1:8 $17.32 \mathrm{~nm}$ AgNPs. (b) Cap-LC chromatogram for 1:8 $17.32 \mathrm{~nm}$ AgNPs

\subsection{6 nm Sample} S7).

Synthesis was characterized by TEM (Figure S6), UV-Vis and Cap-LC (Figure

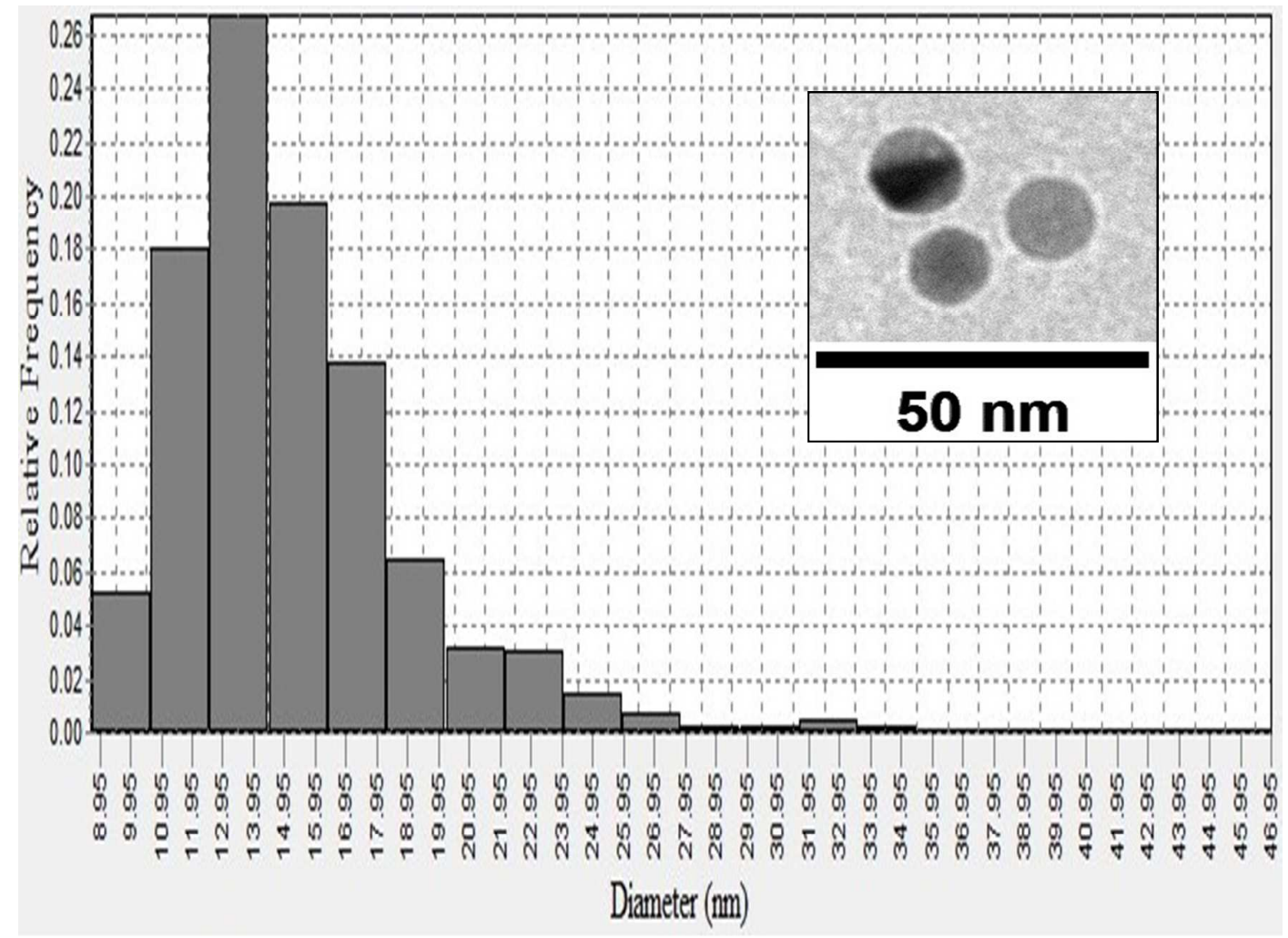

Figure S6. TEM histogram of $15.36 \mathrm{~nm}$ AgNPs showing their size distribution. Up-right: TEM picture. 
a)

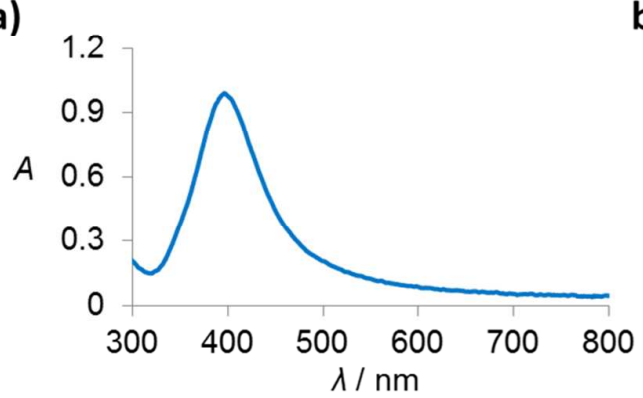

b)

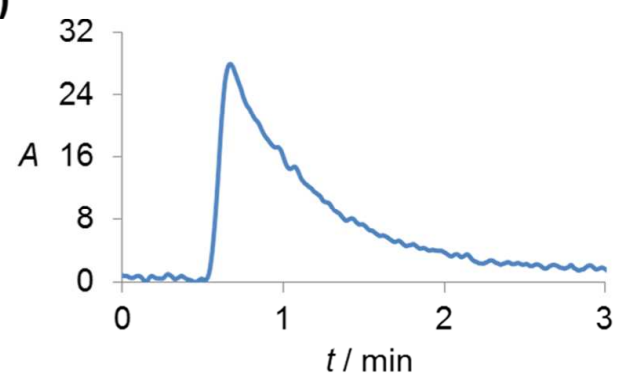

Figure S7. (a) UV-Vis spectrum of 1:4 $15.36 \mathrm{~nm}$ AgNPs. (b) Cap-LC chromatogram for 1:8 $15.36 \mathrm{~nm}$ AgNPs

\section{$12.23 \mathrm{~nm}$ Sample}

Synthesis was characterized by TEM (Figure S8), UV-Vis and Cap-LC (Figure S9).

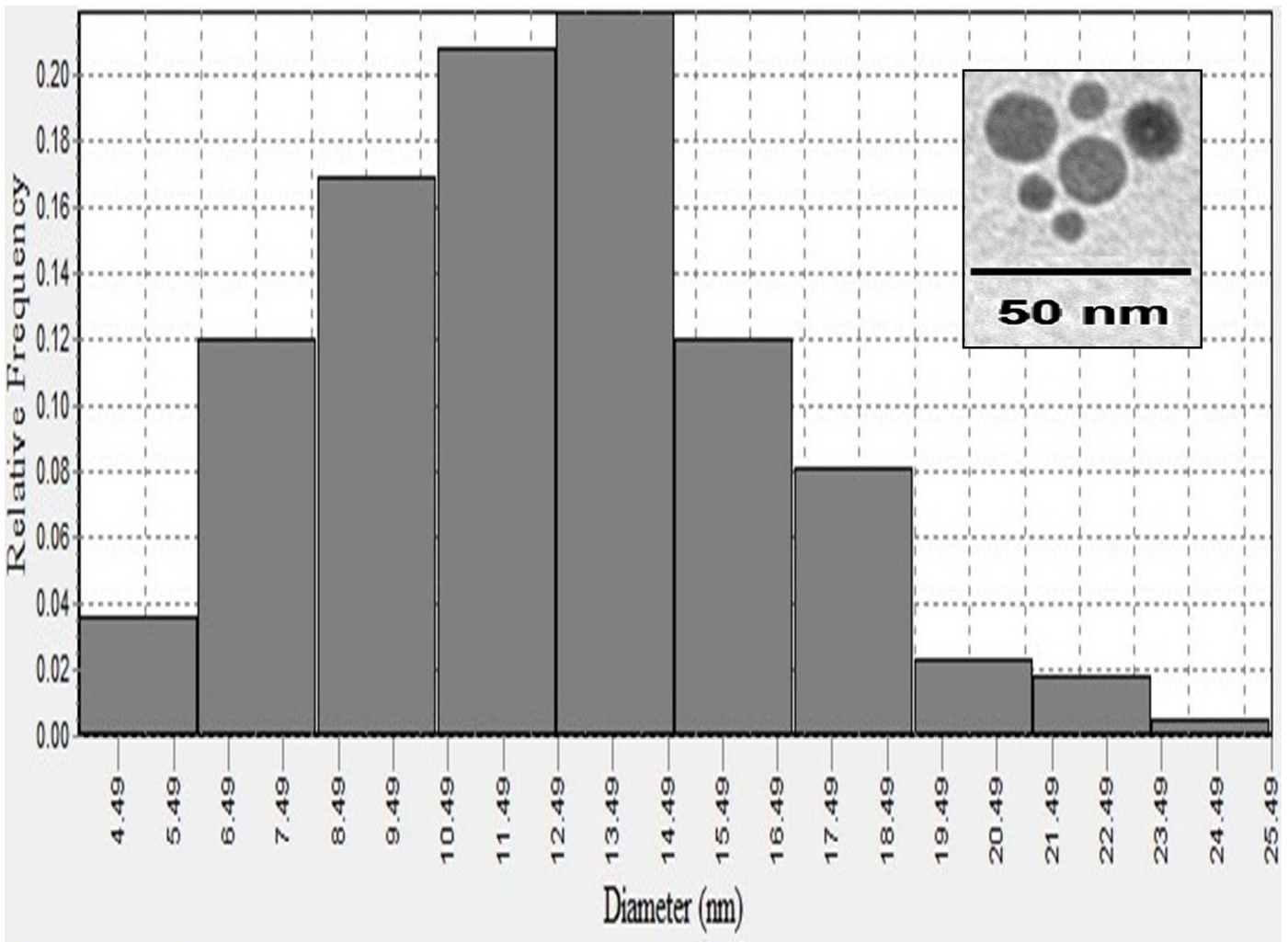

Figure S8. TEM histogram of $12.23 \mathrm{~nm}$ AgNPs showing their size distribution. Up-right: TEM picture. 
a)

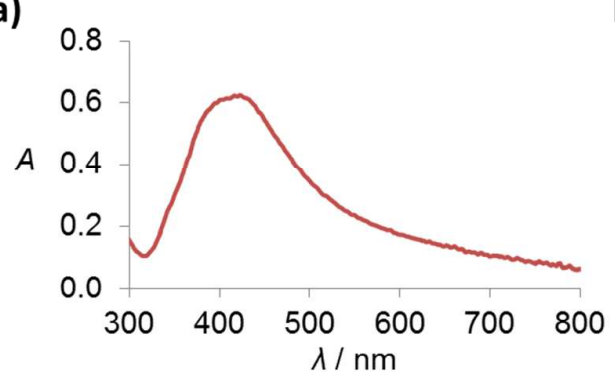

b)

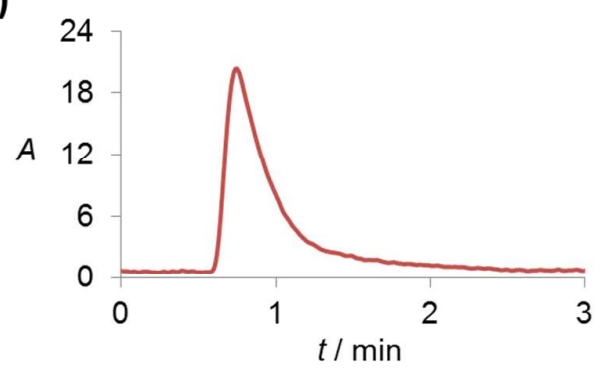

Figure S9. (a) UV-Vis spectrum of 1:8 12.23 nm AgNPs. (b) Cap-LC chromatogram for 1:16 $12.23 \mathrm{~nm}$ AgNPs

\section{$13.92 \mathrm{~nm}$ Sample}

Synthesis was characterized by TEM (Figure S10), UV-Vis and Cap-LC (Figure S11).

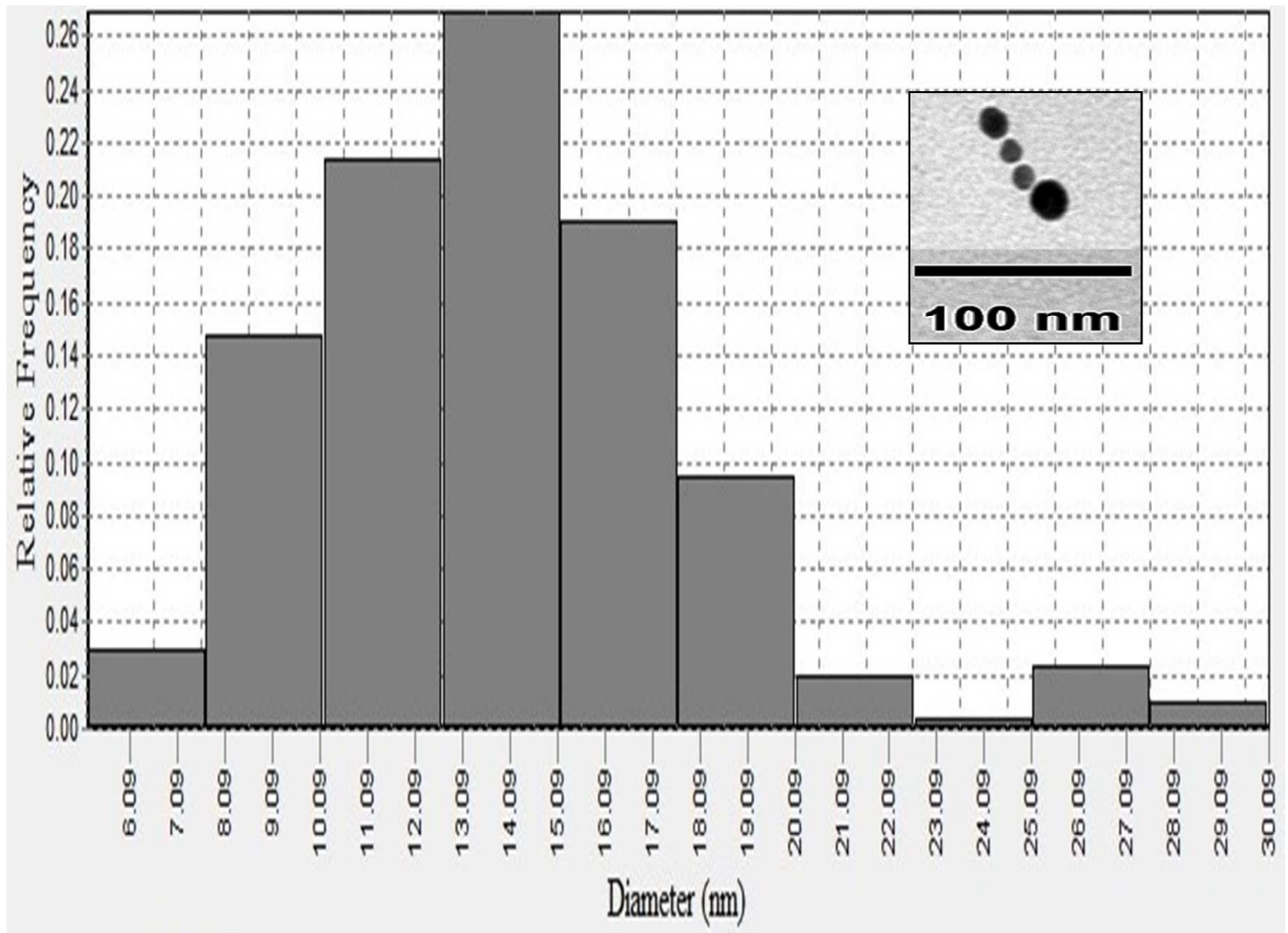

Figure S10. TEM histogram of $13.92 \mathrm{~nm} \mathrm{AgNPs} \mathrm{showing} \mathrm{their} \mathrm{size} \mathrm{distribution.} \mathrm{Up-right:} \mathrm{TEM} \mathrm{picture.}$ 
a)

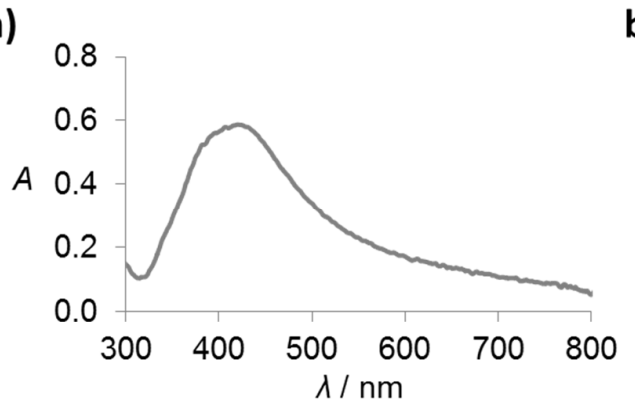

b)

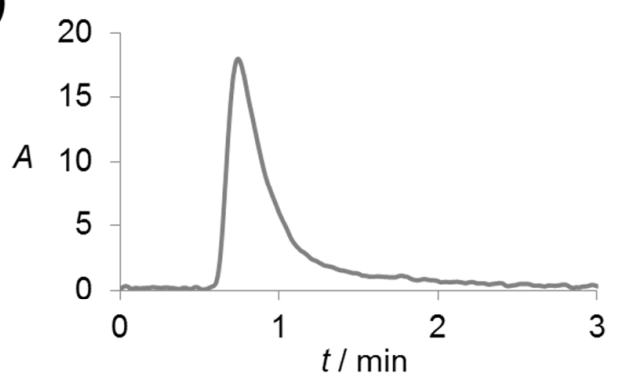

Figure S11. (a) UV-Vis spectrum of 1:8 13.92 nm AgNPs. (b) Cap-LC chromatogram for 1:16 $13.92 \mathrm{~nm}$ AgNPs

\section{$16.19 \mathrm{~nm}$ Sample} S13).

Synthesis was characterized by TEM (Figure S12), UV-Vis and Cap-LC (Figure

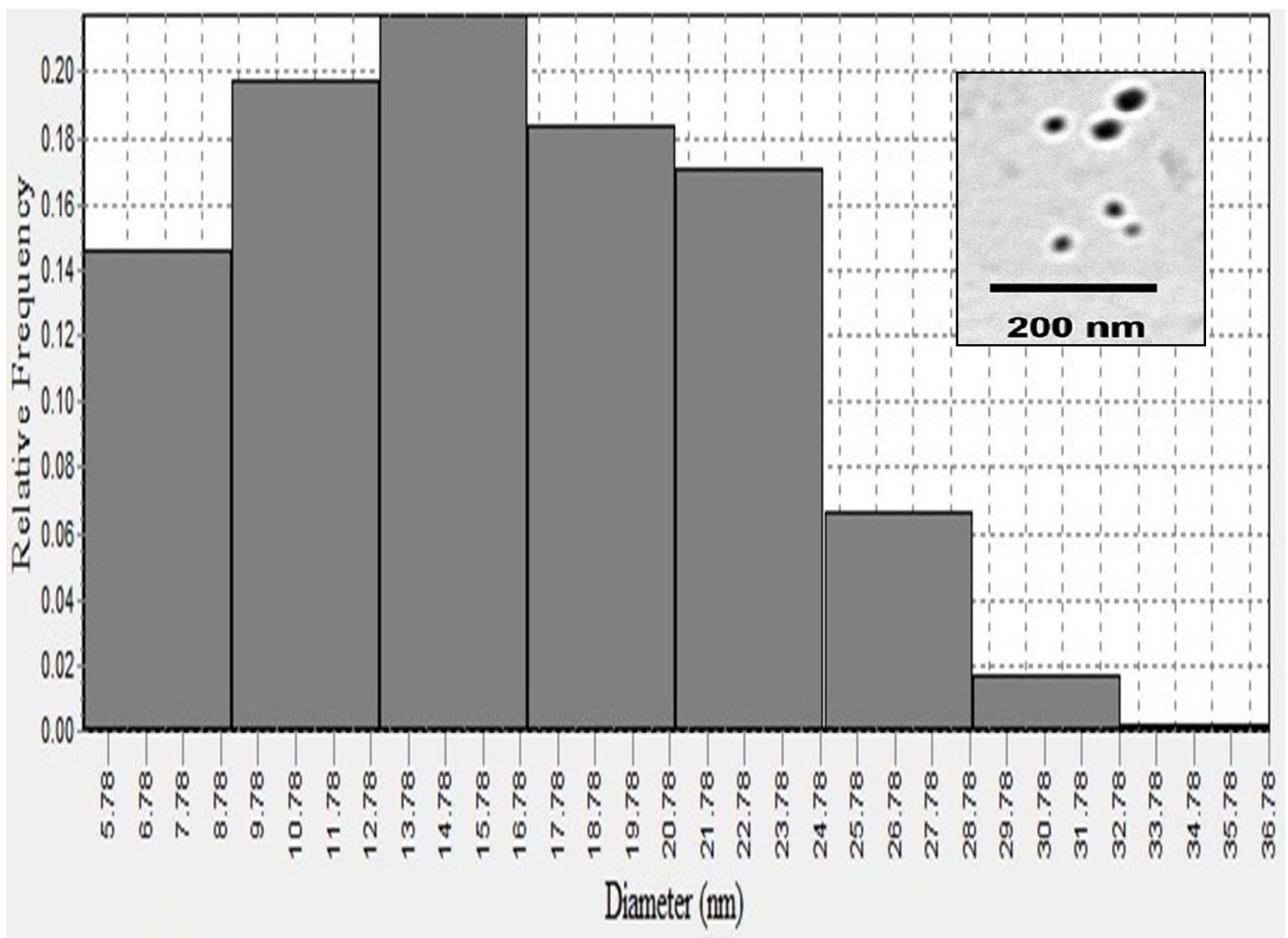

Figure S12. TEM histogram of $16.19 \mathrm{~nm}$ AgNPs showing their size distribution. Up-right: TEM picture. 

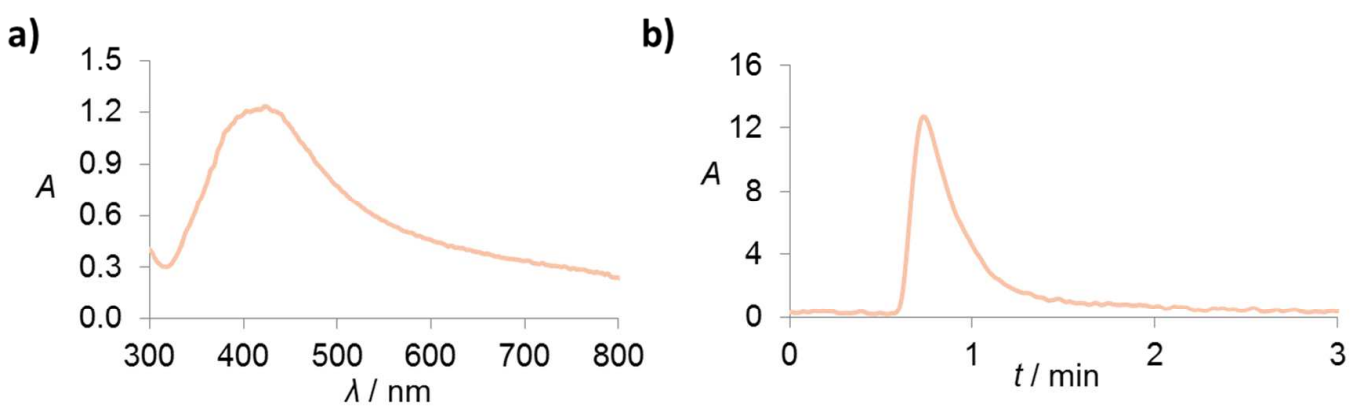

Figure S13. (a) UV-Vis spectrum of 1:8 $16.19 \mathrm{~nm}$ AgNPs. (b) Cap-LC chromatogram for 1:16 16.19 nm AgNPs

\section{$25.57 \mathrm{~nm}$ Sample}

Synthesis was characterized by TEM (Figure S14), UV-Vis and Cap-LC (Figure $\mathrm{S} 15)$.

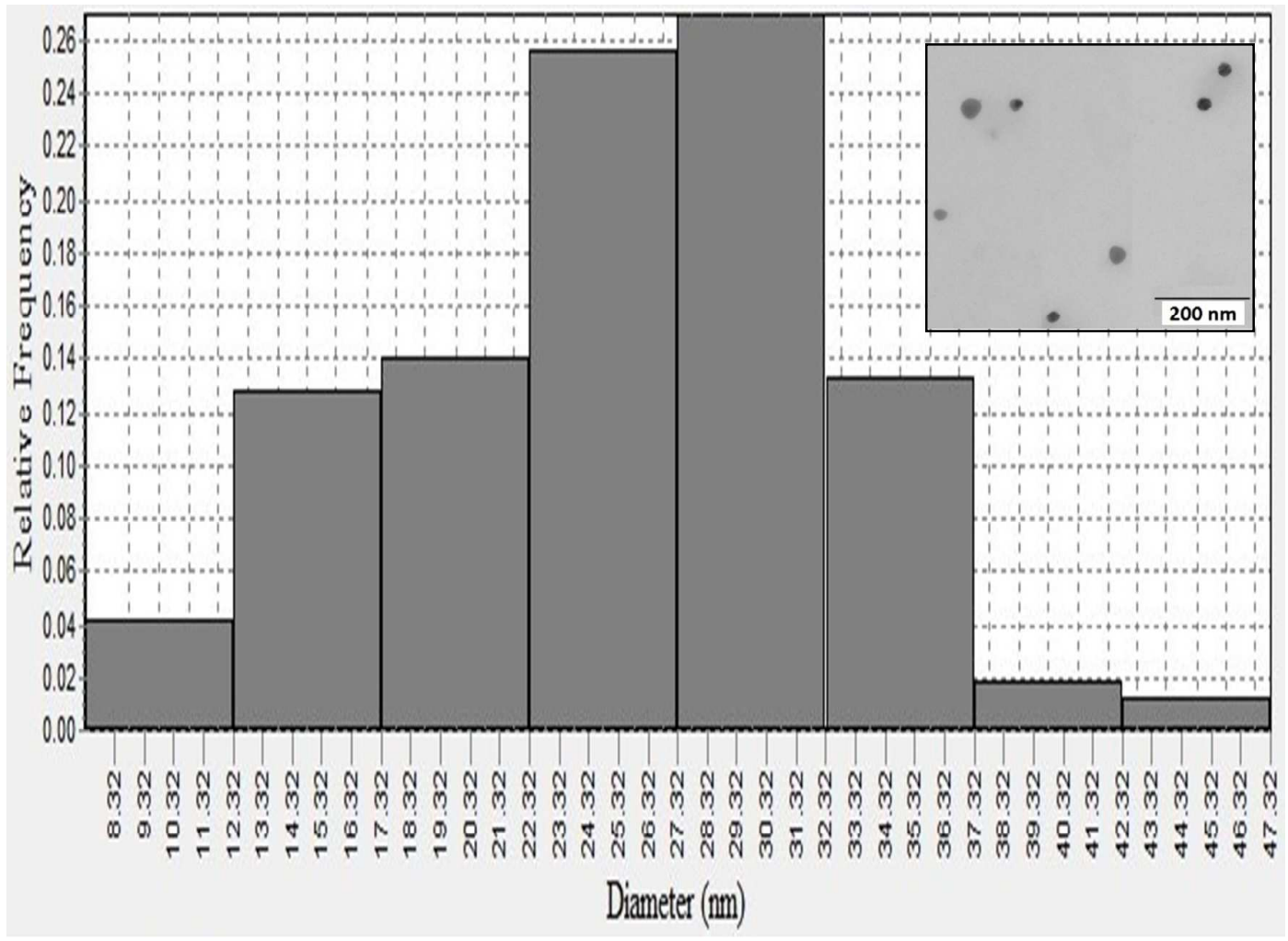

Figure S14. TEM histogram of $25.57 \mathrm{~nm}$ AgNPs showing their size distribution. Up-right: TEM picture. 
a)

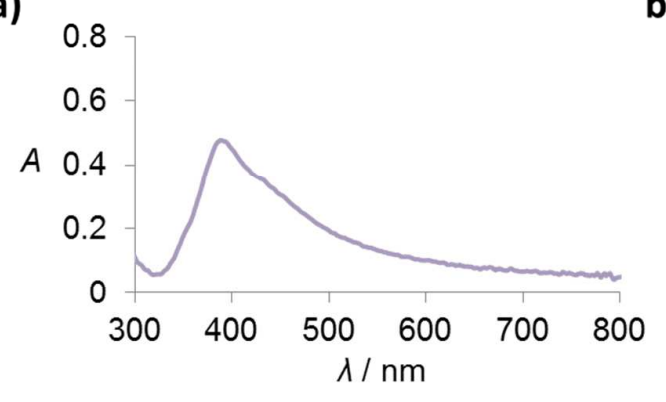

b)

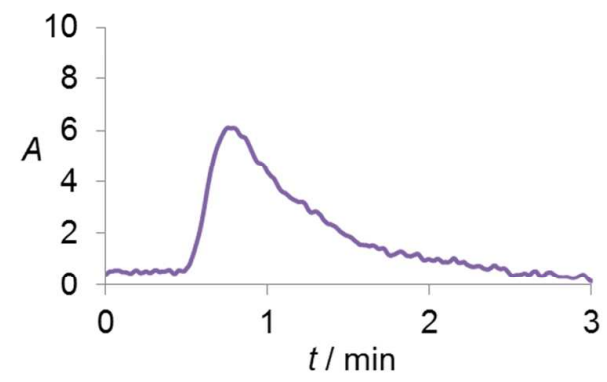

Figure S15. (a) UV-Vis spectrum of 1:8 $25.57 \mathrm{~nm}$ AgNPs. (b) Cap-LC chromatogram for 1:8 $25.57 \mathrm{~nm}$ AgNPs

\subsection{5 nm Sample} S17).

Synthesis was characterized by TEM (Figure S16), UV-Vis and Cap-LC (Figure

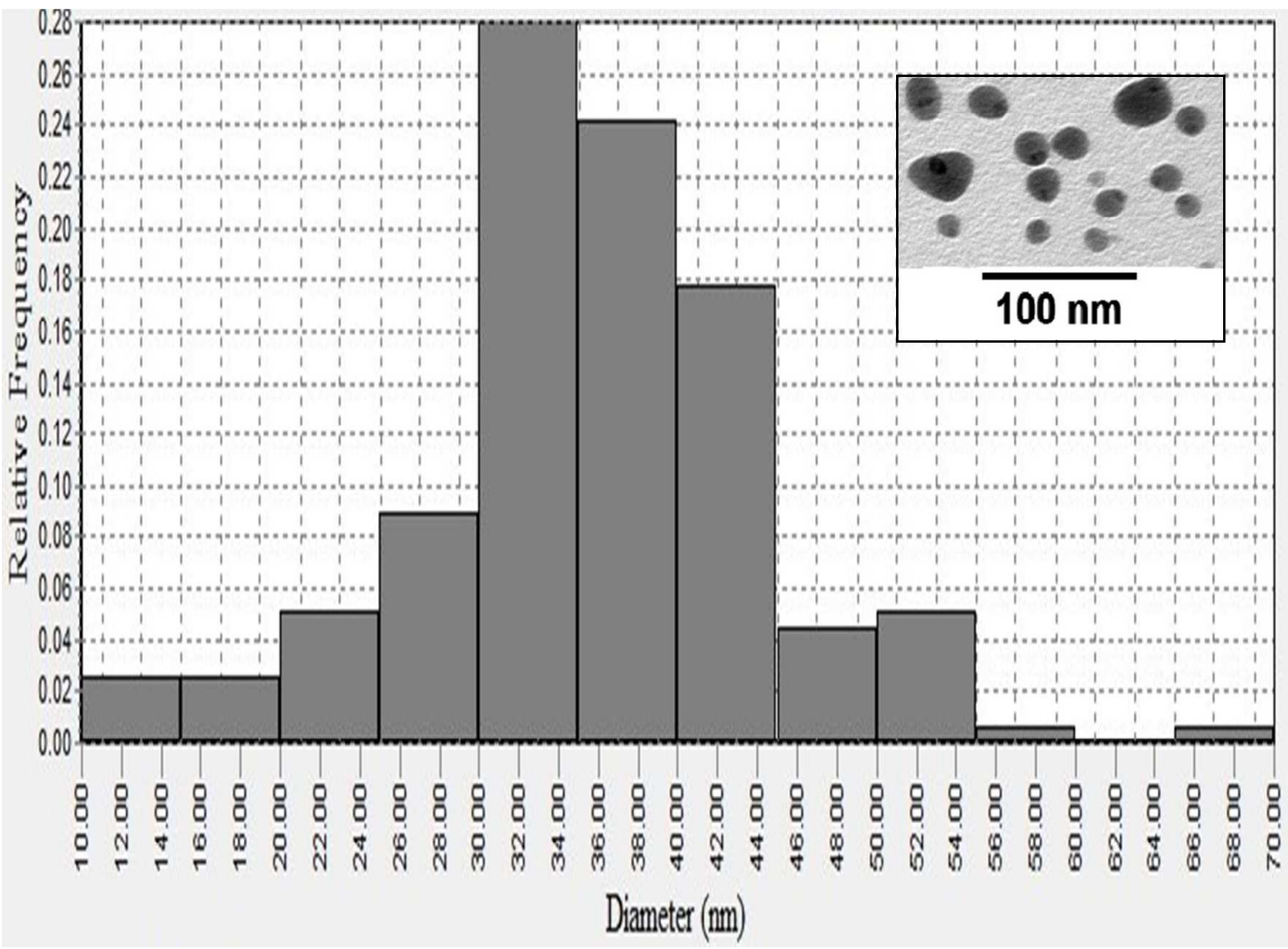

Figure S16. TEM histogram of $38.15 \mathrm{~nm}$ AgNPs showing their size distribution. Up-right: TEM picture. 
a)

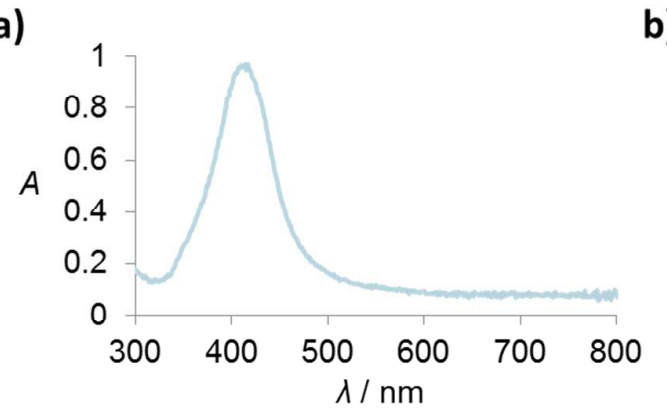

b)

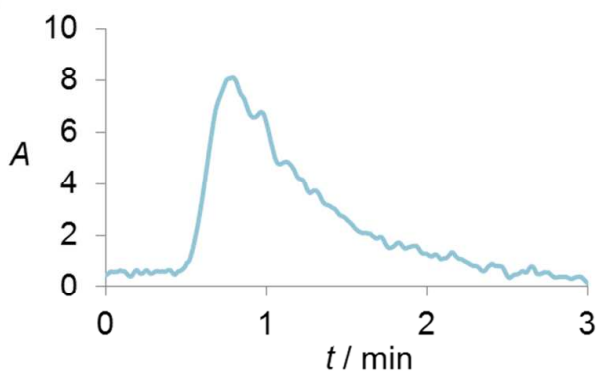

Figure S17. (a) UV-Vis spectrum of 1:15 $38.15 \mathrm{~nm}$ AgNPs. (b) Cap-LC chromatogram for 1:2 $38.15 \mathrm{~nm}$ AgNPs

\section{Additional samples for reproducibility studies:}

14.82 nm Sample. Synthesis characterization is shown in Figure S18.
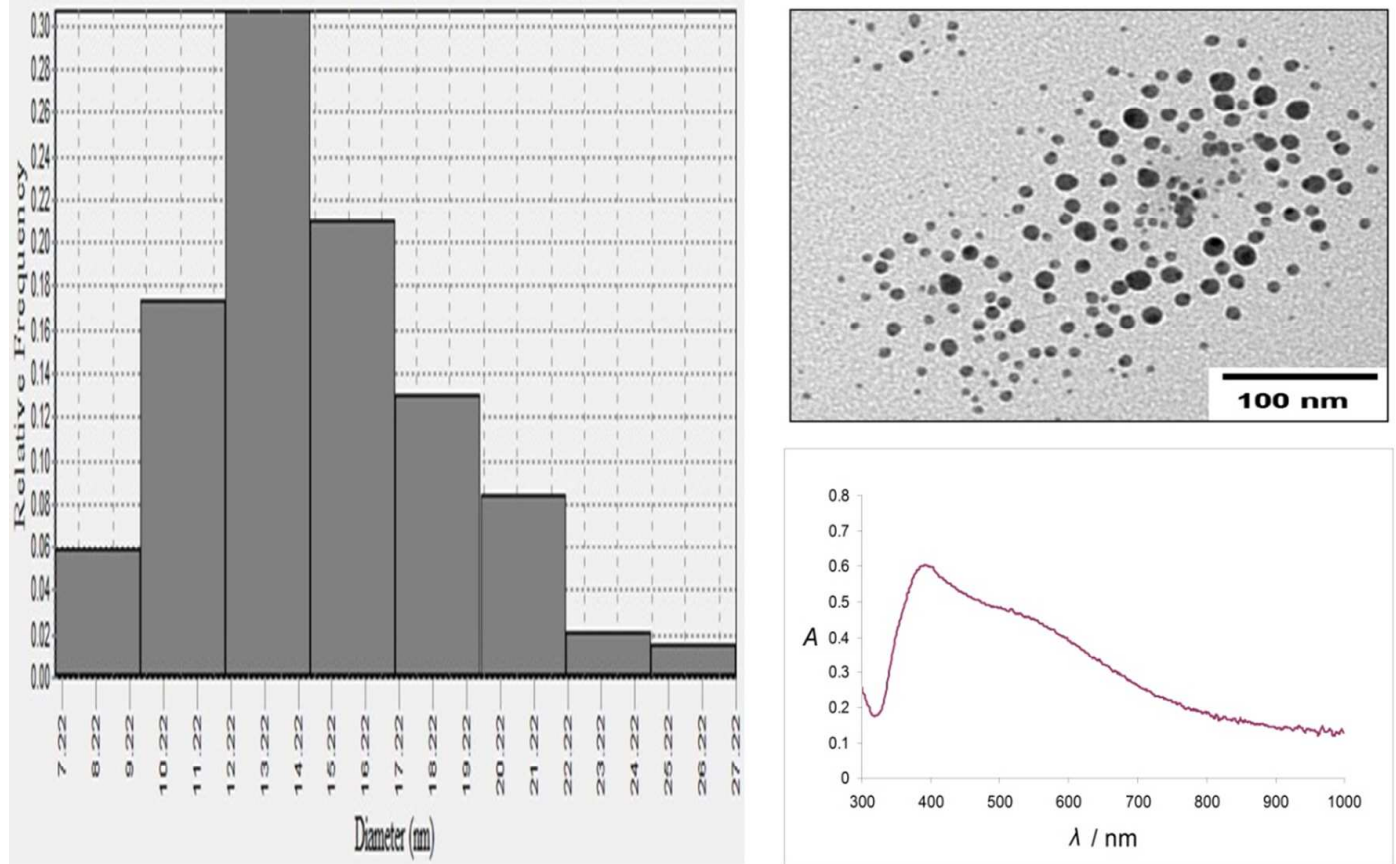

Figure S18. Left: TEM histogram showing size distributions. Up-Right: TEM image of $14.82 \mathrm{~nm}$ AgNPs. Down-Right: UV-Vis spectrum of 1:16 14.82 nm AgNPs. 
15.1 nm Sample. Synthesis characterization is shown in Figure S19.
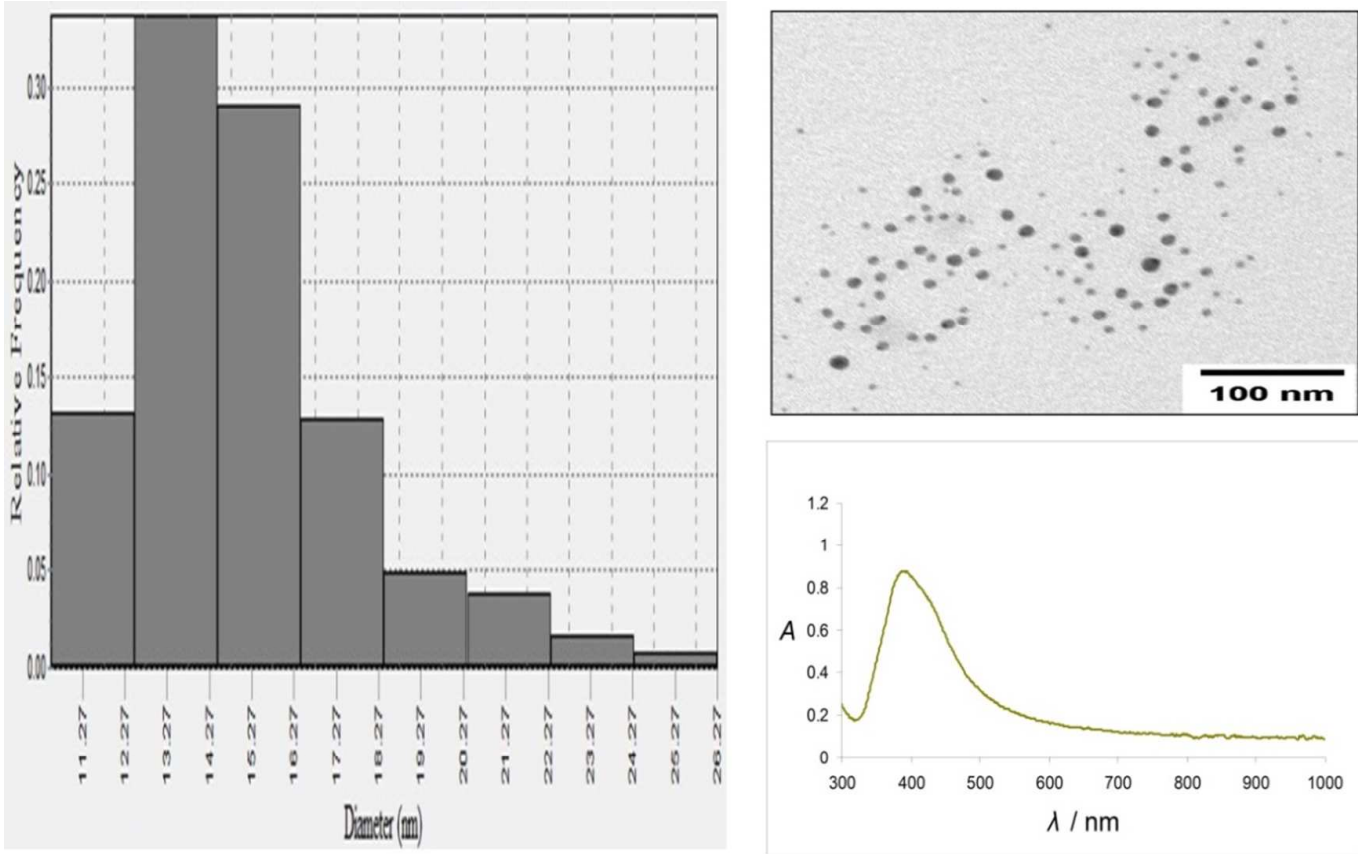

Figure S19. Left: TEM histogram showing size distributions. Up-Right: TEM image of $15.1 \mathrm{~nm}$ AgNPs. Down-Right: UV-Vis spectrum of 1:16 15.1 nm AgNPs.

\section{Section 8. Non-spherical AgNPs and stability studies by Cap-LC}

Following the $\mathrm{KBr}$ based synthesis procedure of AgNPs (described in Section 1), triangular-shaped NPs were obtained. The AgNP dispersions containing these nanoprisms are blue. The detection wavelength was changed to $600 \mathrm{~nm}$ corresponding to the wavelength of maximum absorbance. 1:8 dilutions of triangular-shaped AgNP synthesis were injected; results are shown in Figure S20 (Cap-LC chromatogram, UVVis spectrum and DAD spectrum as well as pictures are displayed in the figure). This experiment shows that nanoprisms could also be analyzed.

On the other hand, considering that NPs are subject to stress through the chromatographic system, whether unstable or degraded synthesis would respond or not using the developed Cap-LC method is still unknown. Up until the time of this writting, all the tested samples have not been damaged through time.

Under the same conditions in which $15.36 \mathrm{~nm}$ AgNP (previously used) synthesis was made, a new synthesis was prepared but this time adding $5 \% \mathrm{w} / \mathrm{v}$ sodium citrate (five times more). This reagent has a key role during the process: to be the secondary reductant and stabilizing agent [9]. It can be expected that NPs will be larger as more citrate can stabilize the particle structure. TEM analysis confirmed this prediction as the new AgNP synthesis had an average particle diameter of $16.72 \mathrm{~nm}$ (S2, Figure S21a). 15.36 and $16.72 \mathrm{~nm} \mathrm{AgNPs}$ were tested in Cap-LC. Chromatograms are shown in Figure S21b. S2 did not respond properly in the proposed method (the predicted S2 
$\mathrm{d}_{\mathrm{AgNPs}}$ is larger than the one calculated by TEM). For establishing preliminary conclusions on this matter, additional tests were performed on both samples.
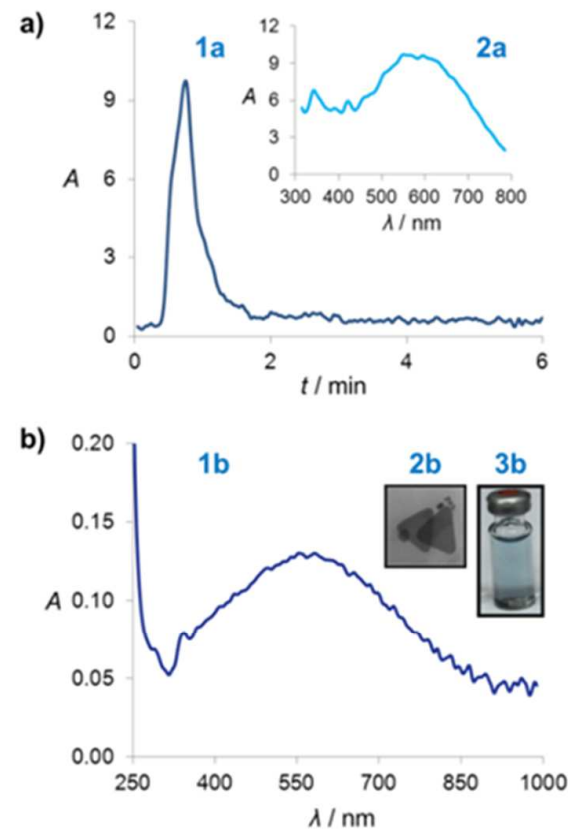

Figure S20. Non-spherical AgNP studies. (a) Cap-LC results: (1a) chromatogram at $600 \mathrm{~nm}$ for 1:8 AgNPs and (2a) DAD spectrum. (b) Additional data obtained: (1b) UV-Vis spectrum for 1:8 AgNPs, (2b) TEM and (2c) vial pictures of the studied non-spherical AgNPs.

It has been stated that AgNPs are stable in ethanol [10]. We used this fact to corroborate the stability (in a given organic solvent) for both syntheses, which were diluted (1:16) in water (test blank) and in ethanol. UV-Vis spectra were registered. $16.72 \mathrm{~nm}$ AgNPs SPRB was disrupted, while for $15.36 \mathrm{~nm}$ AgNPs this band remained unchanged for both solvents. Results are displayed in figure S21c. This experiment corroborates the instability observed in Cap-LC for the second synthesis.

We also assayed a functionality-related property by using a recent application of AgNPs: these particles can be used for analyzing quaternary ammonium surfactants in a colorimetric method [11]. Both samples (1:8 dilutions) were tested for detecting benzalkonium chloride (BZKC). Additions of increasing concentrations of BZKC (here in a working range from 0 to $112 \mu \mathrm{M}$ ) should induce aggregation of AgNPs (shift from yellow-orange to colorless) through the hydrophobic effect in acidic media (using diluted nitric acid). AgNP aggregation has to be gradual: if BZKC concentration increases, the hydrophobic effect and the NP aggregation are also increased. For 15.36 $\mathrm{nm}$ AgNPs, this tendency was verified. However, for $16.72 \mathrm{~nm}$ AgNPs the aggregation did not increase gradually (absorbance increased and decreased randomly). In other words, a synthesis of AgNP which is unstable in organic solvents and does not respond in the Cap-LC method cannot detect properly a quaternary ammonium surfactant. Results are displayed in Figure S21d.

To conclude, it can be said that Cap-LC gives stability-related information about AgNP syntheses (e.g. solvent stability and functionality). These parameters are difficult to quantify by microscopic techniques. 
a)

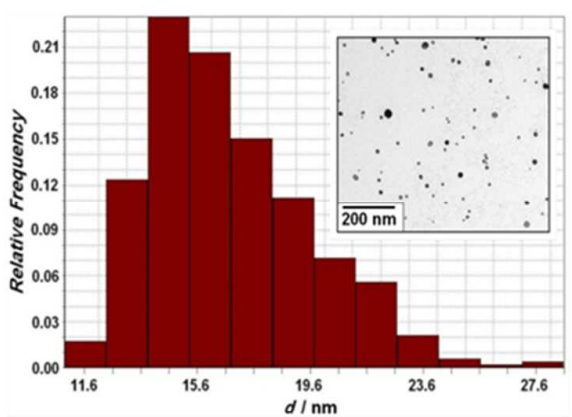

b)

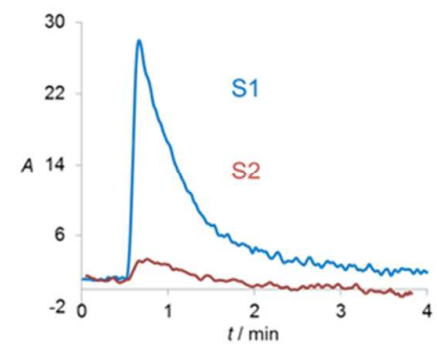

c)

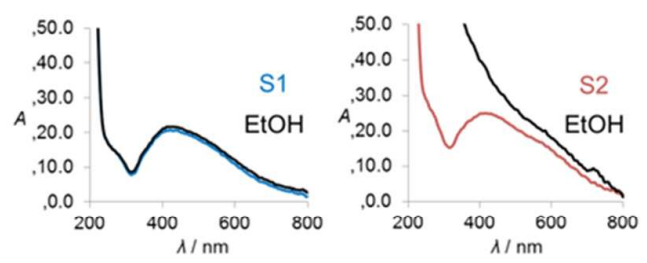

d)

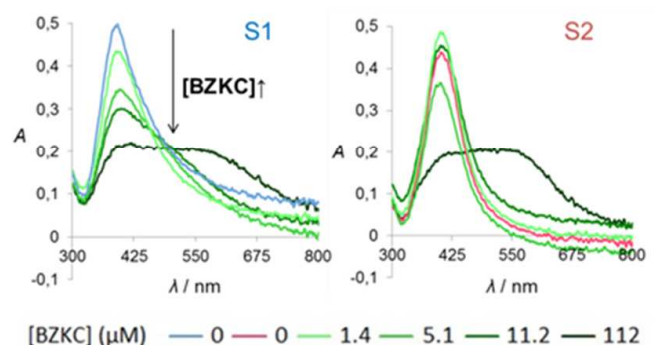

Figure S21. Syntheses stability-related studies. (a) TEM analysis: histogram of particle distribution and TEM pictures of $16.72 \mathrm{~nm}$ AgNPs (S2). (b) Cap-LC results for 1:8 diluted $15.36 \mathrm{~nm}$ (S1) and $16.72 \mathrm{~nm}$ (S2) AgNPs. UV-Vis experiments: (c) S1 and S2 spectra obtained for 1:16 dilutions in water (blue or red line) and ethanol (black line) and (d) S1 and S2 spectra obtained for series of samples in which BZKC was added from 0 (blue or red line) to $112 \mu \mathrm{M}$ (series from lighter to darker green lines). For S1, the expected variation tendency was verified and for S2 no tendency was obtained.

\section{Section 9. IT-SPME-Cap-LC}

IT-SPME-Cap-LC manifold, preliminary studies and optimization. Figure S22 shows the device employed. The IT-SPME was achieved by coupling a PDMS capillary column to the injection valve. Also, the chromatograms obtained using different PDMS capillaries (TRB-5 and TRB-35) are displayed. The PDMS TRB-35 column provided the best results in terms of intensity and peak separation for AgNP aqueous dispersions. 
a)

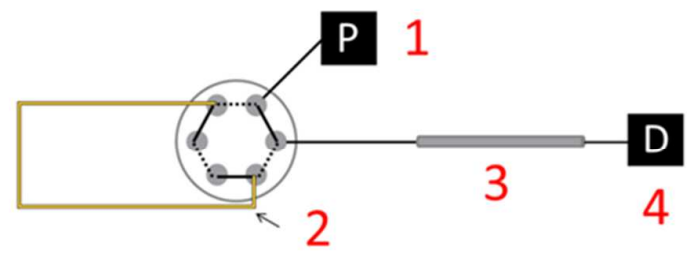

b)

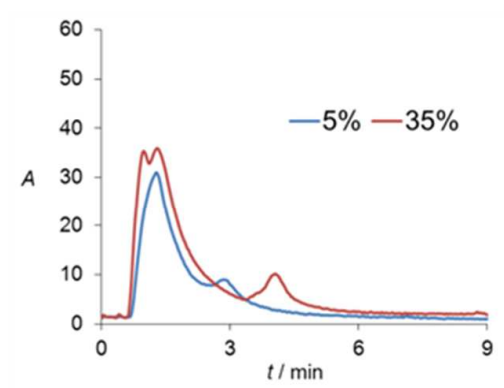

Figure S22. IT-SPME optimization. (a) IT-SPME-Cap-LC manifold: (1) Pump working at isocratic mode, (2) PDMS capillary column coupled to the system injection valve, (3) Jupiter C18 analytical column and (4) DAD detector. (b) Chromatograms obtained using different adsorbent phases with a 35 $\mathrm{cm}$ long capillary column.

The chromatogram for $60 \mu \mathrm{L}$ of water in IT-SPME with a $70 \mathrm{~cm}$ long PDMSTRB 35 capillary is shown in Figure S23. The later peak of the chromatogram corresponds to an interfering compound (its DAD spectrum is shown in the figure).

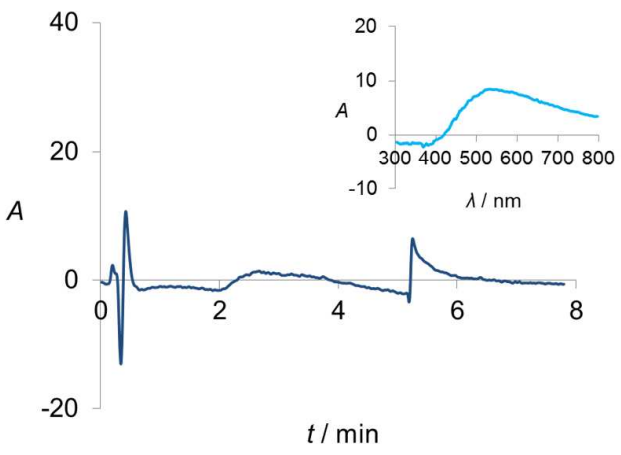

Figure S23. Chromatogram of the blank (loop filled with water) and DAD spectrum of the later peak (upright) using a $70 \mathrm{~cm}$ long PDMS 35 capillary.

IT-SPME-Cap-LC calibration. The IT-SPME-Cap-LC chromatograms of the different levels of concentration as well as the calibration curve are displayed in Figures S24 and $\mathrm{S} 25$. The sum of the two peaks obtained was used as analytical signal. The $17.32 \mathrm{~nm}$ AgNP synthesis was selected as standard. 


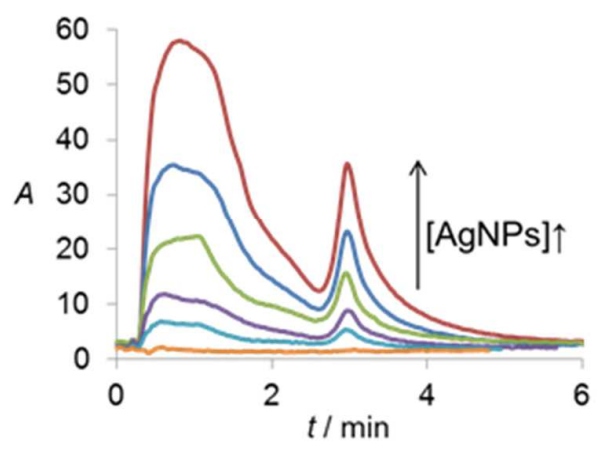

Figure S24. IT-SPME-Cap-LC calibration results. Chromatograms obtained for a blank and five 17.32 $\mathrm{nm}$ AgNP levels of concentration within 0-0.33 nM (increasing as the arrow shows).

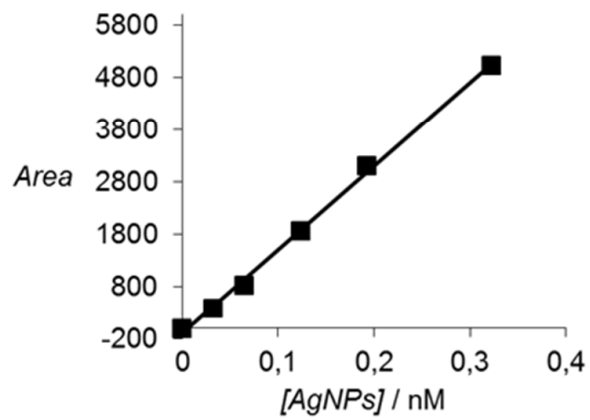

Figure S25. Calibration curve representation for the IT-SPME-Cap-LC method using the $17.32 \mathrm{~nm}$ synthesis as standard.

IT-SPME-Cap-LC AuNP mixture. The IT-SPME-Cap-LC chromatograms of an experiment to analyze a mixture of 5 and $20 \mathrm{~nm}$ AuNPs are displayed in Figure S26. The calculated ratio for $10 \mu \mathrm{L}$ injections were $0.42,0.13$ and 0.79 for the mixture, 5 and $20 \mathrm{~nm}$ respectively. The peak ratio of the mixture was an intermediate value suggesting that NPs interact between them. Particle redistribution and water polarization occurred.

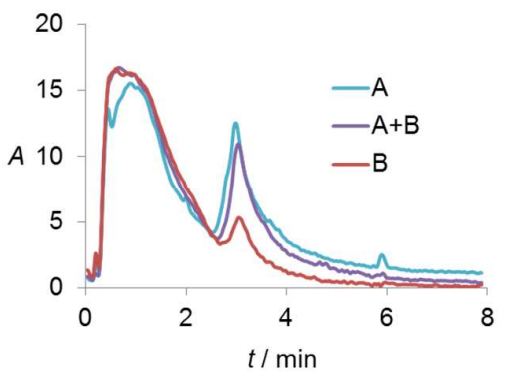

Figure S26. Obtained chromatograms at $530 \mathrm{~nm}$ for standard A (1:8 $20 \mathrm{~nm}$ AuNPs), standard B (1:16 5 $\mathrm{nm}$ AuNPs) and mixture A+B (1:8 $20 \mathrm{~nm}$ AuNPs and 1:16 $5 \mathrm{~nm}$ AuNPs). 


\section{Section 10. Syntheses prepared for DLS measurements}

Figure S27 shows both TEM histogram and IT-SPME-Cap-LC chromatogram for the two samples (1 and 2) measured by DLS.
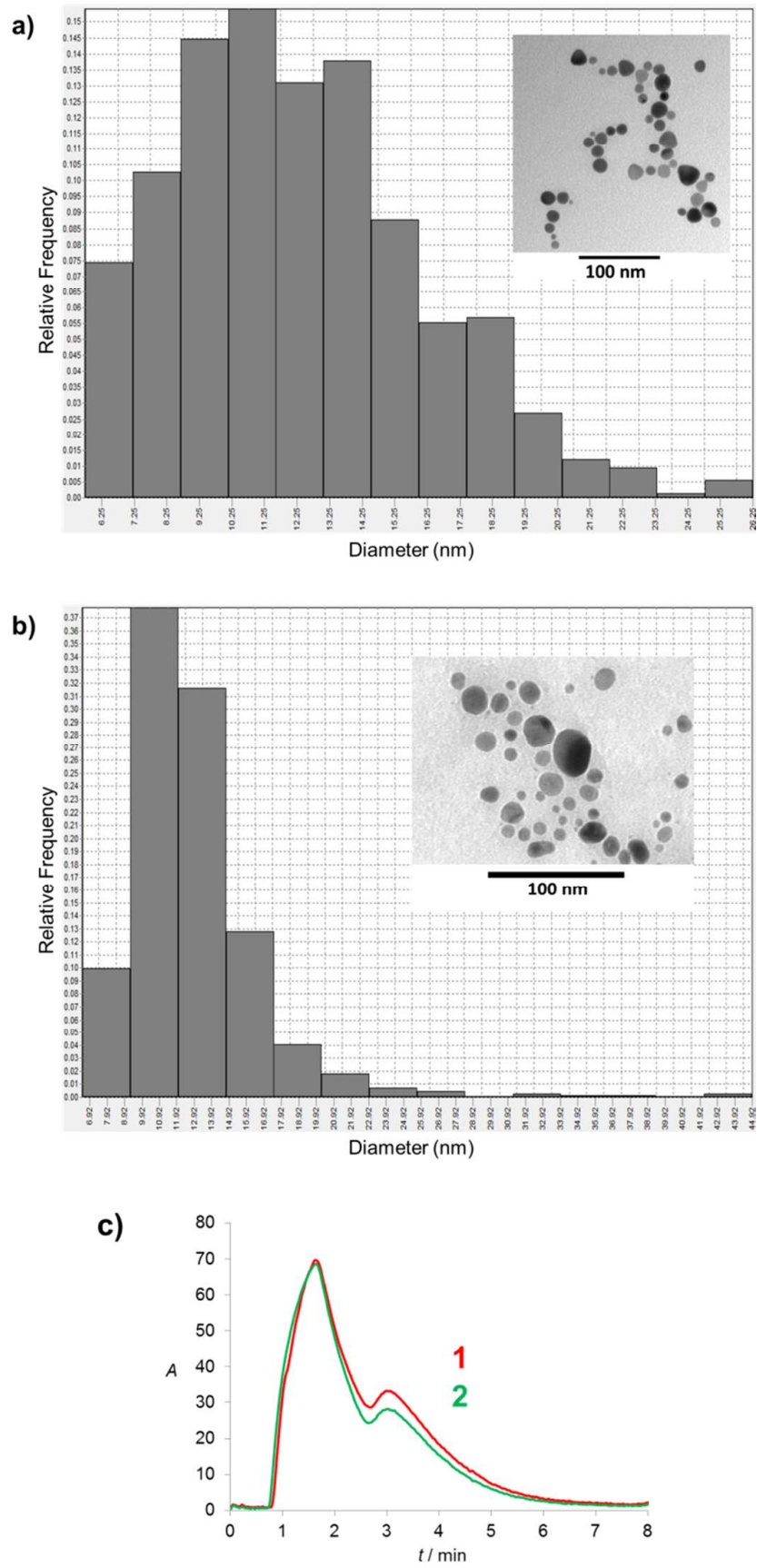

Figure S27. (a) TEM histogram of $12.34 \mathrm{~nm} \mathrm{AgNPs} \mathrm{showing} \mathrm{their} \mathrm{size} \mathrm{distribution.} \mathrm{Up-right:} \mathrm{TEM}$ picture. (b) TEM histogram of $12.83 \mathrm{~nm}$ AgNPs showing their size distribution. Up-right: TEM picture. (c)IT-SPME-Cap-LC chromatograms for: (1) 1:16 $12.34 \mathrm{~nm}$ and (2) 1:16 $12.83 \mathrm{~nm}$ AgNPs. 


\section{Section 11. Concentrations (measured and corrected) of the AgNPs samples}

Table S1 shows the interpolated concentrations of AgNPs calculated from the peak areas of the chromatograms and the corrected (or final) concentrations of AgNPs after applying the dilution factor. Although in Table S1 only one dilution per sample can be seen, other dilutions were injected and treated as well.

Table S1. AgNPs syntheses parameters

\begin{tabular}{|c|c|c|c|}
\hline $\begin{array}{c}\text { TEM measured } \\
\text { Diameter }(\mathrm{nm})\end{array}$ & $\begin{array}{c}\text { Cap-LC interpolated } \\
{[\text { AgNPs] }(\mathrm{nM})}\end{array}$ & $\begin{array}{c}\text { Sample } \\
\text { dilution }\end{array}$ & $\begin{array}{c}\text { Cap-LC } \\
{[\text { AgNPs] }(\mathrm{nM})}\end{array}$ \\
\hline 12.23 & 0.57 & $1: 16$ & 9.12 \\
\hline $12.34^{*}$ & 0.25 & $1: 32$ & 7.98 \\
\hline $12.83^{*}$ & 0.23 & $1: 32$ & 7.38 \\
\hline 13.92 & 0.39 & $1: 16$ & 6.24 \\
\hline 15.36 & 0.56 & $1: 8$ & 4.48 \\
\hline 16.19 & 0.30 & $1: 16$ & 4.80 \\
\hline 17.32 & 0.43 & $1: 8$ & 3.45 \\
\hline 25.57 & 0.14 & $1: 8$ & 1.14 \\
\hline 38.15 & 0.22 & $1: 2$ & 0.44 \\
\hline
\end{tabular}

*IT-SPME

\section{References}

[1] González-Fuenzalida, R. A.; Moliner-Martínez, Y.; González-Béjar, M.; MolinsLegua, C.; Verdú-Andrés, J.; Pérez-Prieto, J.; Campins-Falcó, P. Anal. Chem. 2013, 85, 10013-10016.

[2] Frank, A. J.; Cathcart, N.; Maly, K. E.; Kitaev, V. Journal of Chem. 2010, 87, 10981101.

[3] Millstone, J. E.; Hurst, S. J.; Métraux, G. S.; Cutler, J. I.; Mirkin, C. A. Small 2009, $5,646-664$.

[4] Agnihotri, S.; Mukherji, S.; Mukherji, S. RSC Adv. 2014, 86, 3974.

[5] Lee, P. C.; Meisel, D. J. Phys. Chem. 1982, 86, 3391.

[6] Pacioni, N. L.; González-Béjar, M.; Alarcón, E.; McGilvray, K. L.; Scaiano, J. C. J. Am. Chem. Soc. 2010, 132, 6298.

[7] Soto-Alvaredo, J.; Montes-Bayón, M.; Bettmer, J. Anal. Chem. 2013, 85, 13161321.

[8] Tang, B.; Xu, S.; Hou, X.; Li, J.; Sun, L.; Xu, W.; Wang, X. ASC Appl. Mater. Interfaces 2013, 5, 646-653.

[9] Agnihotri S.; Mukherji S.; Mukherji, S. RSC Adv., 2014, 4, 3974-3983.

[10] Tang, B.; Xu, S.; Hou, X.; Li, J.; Sun, L.; Xu, W.; Wang, X. ASC Appl. Mater. Interfaces, 2013, $5,646-653$.

[11] Zheng, L. Q.; Yu, X. D.; Xu, J. J.; Chen, H. Y. Talanta, 2014, 118, 90-95. 